\title{
DOMAIN REPRESENTATIONS INDUCED BY DYADIC SUBBASES
}

\author{
HIDEKI TSUIKI AND YASUYUKI TSUKAMOTO
}

Graduate School of Human and Environmental Studies, Kyoto University, Sakyo-ku, Kyoto, Japan e-mail address: \{tsuiki,tsukamoto\},@i.h.kyoto-u.ac.jp

\begin{abstract}
We study domain representations induced by dyadic subbases and show that a proper dyadic subbase of a second-countable regular space $X$ induces an embedding of $X$ in the set of minimal limit elements of a subdomain $D$ of $\{0,1, \perp\}^{\omega}$. In particular, if $X$ is compact, then $X$ is a retract of the set of limit elements of $D$.
\end{abstract}

\section{INTRODUCTION}

From a computational point of view, it is natural to consider a subbase of a second-countable $T_{0}$ space $X$ as a collection of primitive properties of $X$ through which one can identify each point of $X$. In this way, by fixing a numbering of the subbase, one can represent each point of $X$ as a subset of $\mathbb{N}$ and construct a domain representation of $X$ in the domain $P_{\omega}$ of subsets of $\mathbb{N}$ [3, 17]. Note that $P_{\omega}$ is isomorphic to the domain of infinite sequences of the Sierpinski space $\{1, \perp\}$.

On the other hand, each regular open set $A$ (i.e., an open set which is equal to the interior of its closure) of a topological space $X$ divides $X$ into three parts: $A$, the exterior of $A$, and their common boundary. Therefore, one can consider a pair of regular open subsets which are exteriors of each other as a pair of primitive properties and use a subbase which is composed of such pairs of open sets in representing the space. Such a subbase is called a dyadic subbase and a dyadic subbase of a space $X$ induces a domain representation of $X$ in the domain $\mathbb{T}^{\omega}$ of infinite sequences of $\mathbb{T}=\{0,1, \perp\}$. In [20] and [12], the authors introduced to a dyadic subbase the properness property which expresses a kind of orthogonality between the components and studied domain representations of Hausdorff spaces induced by proper dyadic subbases. In this representation, the domain is fixed to $\mathbb{T}^{\omega}$ and an embedding $\varphi_{S}$ of a Hausdorff space $X$ in $\mathbb{T}^{\omega}$ is derived from a proper dyadic subbase $S$ of $X$.

In this paper, we derive from a dyadic subbase $S$ a domain (i.e., an $\omega$-algebraic pointed dcpo) $D_{S}$ and a bounded complete domain $\widehat{D}_{S}$ which are subdomains of $\mathbb{T}^{\omega}$ containing $\varphi_{S}(X)$ as subspaces. The domain $D_{S}$ has the following properties. (1) If $X$ is a strongly nonadhesive Hausdorff space (Definition 5.4), then the set $L\left(D_{S}\right)$ of limit (i.e., non-compact)

2012 ACM CCS: [Mathematics of computing]: Continuous mathematics-Topology-Point-set topology.

Key words and phrases: domain theory, subbase, compact Hausdorff space.

This work was partially supported by JSPS KAKENHI Grant Number 22500014. 
elements of $D_{S}$ has minimal elements. (2) If $X$ is regular, then $\varphi_{S}$ is an embedding of $X$ in the set of minimal elements of $L\left(D_{S}\right)$. (3) If $X$ is compact, then there is a retraction $\rho_{S}$ from $L\left(D_{S}\right)$ to $X$. That is, every infinite strictly increasing sequence in $K\left(D_{S}\right)$ represents a point of $X$ through $\rho_{S}$ and $\left(D_{S}, L\left(D_{S}\right), \rho_{S}\right)$ is the kind of domain representations studied in [19]. The domain $\widehat{D}_{S}$ also has the properties (1) to (3) and, in addition, it is bounded complete.

We study properties of representations for second-countable Hausdorff spaces and investigate which property holds under each of the above-mentioned conditions. Therefore, a space in this paper means a second-countable Hausdorff space unless otherwise noted. We are mainly interested in the case where $X$ is a regular space because the corresponding domain representations have good properties as we mentioned above. In addition, it is proved in [11] that every second-countable regular space has a proper dyadic subbase and in [12] that every dense-in-itself second-countable regular space has an independent subbase, which is a proper dyadic subbase with an additional property.

We review proper dyadic subbases and their properties in the next section, and we study TTE-representations and domain representations in $\mathbb{T}^{\omega}$ derived from (proper) dyadic subbases in Section 3. We introduce the domains $D_{S}$ and $\widehat{D}_{S}$ in Section 4, and present the strongly nonadhesiveness condition in Section 5 . Then we study domain representations in these domains for the case $X$ is regular in Section 6. Finally, in Section 7, we study the small inductive dimension of the $T_{0}$-spaces $L\left(D_{S}\right)$ and $L\left(\widehat{D}_{S}\right)$ based on a result in [19].

\section{Preliminaries and Notations:}

Bottomed Sequences: Let $\mathbb{N}$ be the set of non-negative integers and $\mathcal{2}$ be the set $\{0,1\}$. Let $\mathbb{T}$ be the set $\{0,1, \perp\}$ where $\perp$ is called the bottom character which means undefinedness. The set of infinite sequences of a set $\Sigma$ is denoted by $\Sigma^{\omega}$. Each element of $\mathbb{T}^{\omega}$ is called a bottomed sequence and each copy of 0 and 1 which appears in a bottomed sequence $p$ is called a digit of $p$. A finite bottomed sequence is a bottomed sequence with a finite number of digits, and the set of all finite bottomed sequences is denoted by $\mathbb{T}^{*}$. We sometimes omit $\perp^{\omega}$ at the end of a finite bottomed sequence and identify a finite bottomed sequence with a finite sequence of $\mathbb{T}$. The set of finite sequences of $\mathcal{Z}$ is denoted by $\mathcal{2}^{*}$.

We define the partial order relation $\sqsubseteq$ on $\mathbb{T}$ by $\perp \sqsubseteq 0$ and $\perp \sqsubseteq 1$, and its product order on $\mathbb{T}^{\omega}$ is denoted by the same symbol $\sqsubseteq$, i.e., for every $p, q \in \mathbb{T}^{\omega}, p \sqsubseteq q$ if $p(n) \sqsubseteq q(n)$ for each $n \in \mathbb{N}$. Then $2^{\omega}$ is the set of maximal elements of $\mathbb{T}^{\omega}$. We consider the $T_{0}$-topology $\{\varnothing,\{0\},\{1\},\{0,1\}, \mathbb{T}\}$ on $\mathbb{T}$, and its product topology on $\mathbb{T}^{\omega}$. We write $\operatorname{dom}(p)=\{k: p(k) \neq$ $\perp\}$ for $p \in \mathbb{T}^{\omega}$. For a finite bottomed sequence $e \in \mathbb{T}^{*}$, the length $|e|$ of $e$ is the maximal number $n$ such that $e(n-1) \neq \perp$. We denote by $\left.p\right|_{n}$ the finite bottomed sequence with $\operatorname{dom}\left(\left.p\right|_{n}\right)=\operatorname{dom}(p) \cap\{0,1, \ldots, n-1\}$ such that $\left.p\right|_{n} \sqsubseteq p$. That is, $\left.p\right|_{n}(k)=p(k)$ if $k<n$ and $\left.p\right|_{n}(k)=\perp$ if $k \geqslant n$. Note that the notation $\left.p\right|_{n}$ is used with a different meaning in [20].

The letters $a$ and $b$ will be used for elements of $\mathcal{L}, c$ for elements of $\mathbb{T}, i, j, k, l, m, n$ for elements of $\mathbb{N}, p$ and $q$ for bottomed sequences, and $d$ and $e$ for finite bottomed sequences. We write $c^{\omega}=(c, c, \cdots) \in \mathbb{T}^{\omega}$ for $c \in \mathbb{T}$. We denote by $p[n:=a]$ the bottomed sequence $q$ such that $q(n)=a$ and $q(i)=p(i)$ for $i \neq n$.

Topology: Throughout this paper, $X$ denotes a second-countable Hausdorff space unless otherwise noted. Therefore, if $X$ is regular, then $X$ is separable metrizable by Urysohn's metrization theorem. Recall that a subset $U$ of $X$ is regular open if $U$ is the interior of its closure. 
A filter $\mathfrak{F}$ on the space $X$ is a family of subsets of $X$ with the following properties.

(1) $\varnothing \notin \mathfrak{F}$.

(2) If $A \in \mathfrak{F}$ and $A \subseteq A^{\prime} \subseteq X$, then $A^{\prime} \in \mathfrak{F}$.

(3) If $A, B \in \mathfrak{F}$, then $A \cap B \in \mathfrak{F}$.

Let $\mathfrak{V}(x)$ denote the family of neighbourhoods of $x \in X$. For a filter $\mathfrak{F}$ on $X$ and a point $x \in X$, if we have $\mathfrak{V}(x) \subseteq \mathfrak{F}$ then we say that $\mathfrak{F}$ converges to $x$.

A family $\mathfrak{B}$ of subsets of $X$ is called a filter base if it satisfies $\varnothing \notin \mathfrak{B}, \mathfrak{B} \neq \varnothing$, and that for all $A, B \in \mathfrak{B}$ there exists $C \in \mathfrak{B}$ such that $C \subseteq A \cap B$. A filter generated by a filter base $\mathfrak{B}$ is defined as the minimum filter containing $\mathfrak{B}$. We say that a filter base converges to $x \in X$ if it generates a filter which converges to $x$.

We denote by $\operatorname{cl}_{Y} A, \operatorname{bd}_{Y} A$, and $\operatorname{ext}_{Y} A$ the closure, boundary, and exterior of a set $A$ in a space $Y$, respectively, and we omit the subscript if the space is obvious.

Domain Theory: Let $(P, \sqsubseteq)$ be a partially ordered set (poset). We say that two elements $p$ and $p^{\prime}$ of a poset $P$ are compatible if $p \sqsubseteq q$ and $p^{\prime} \sqsubseteq q$ for some $q \in P$, and write $p \uparrow p^{\prime}$ if $p$ and $p^{\prime}$ are compatible. For $p \in P$ and $A \subseteq P$, we define $\uparrow p=\{q: q \sqsupseteq p\}$, $\downarrow p=\{q: q \sqsubseteq p\}, \uparrow A=\cup\{\uparrow q: q \in A\}$, and $\downarrow A=\cup\{\downarrow q: q \in A\}$. Therefore, we have $\downarrow \uparrow p=\{q: q \uparrow p\}$. We say that $A$ is downwards-closed if $A=\downarrow A$, and upwards-closed if $A=\uparrow A$.

A subset $A$ of a poset $P$ is called directed if it is nonempty and each pair of elements of $A$ has an upper bound in $A$. A directed complete partial order (dcpo) is a partially ordered set in which every directed subset $A$ has a least upper bound (lub) $\sqcup A$. A dcpo is pointed if it has a least element.

Let $(D, \sqsubseteq)$ be a dcpo. A compact element of $D$ is an element $d \in D$ such that for every directed subset $A$, if $d \sqsubseteq \sqcup A$ then $d \in \downarrow A$. An element of $D$ is called a limit element if it is not compact. We write $K(D)$ for the set of compact elements of $D$, and $L(D)$ for the set of limit elements of $D$.

For $x \in D$, we define $K_{x}=K(D) \cap \downarrow x$. A depo $D$ is algebraic if $K_{x}$ is directed and $\sqcup K_{x}=x$ for each $x \in D$, and it is $\omega$-algebraic if $D$ is algebraic and $K(D)$ is countable. In this paper, a domain means an $\omega$-algebraic pointed dcpo. The Scott topology of a domain $D$ is the topology generated by $\{\uparrow d: d \in K(D)\}$. In this paper, we consider a domain $D$ as a topological space with the Scott topology. A poset is bounded complete if every subset which has an upper bound also has a least upper bound. $\mathbb{T}^{\omega}$ is a bounded complete domain such that $K\left(\mathbb{T}^{\omega}\right)=\mathbb{T}^{*}$.

An ideal of a poset $P$ is a directed downwards-closed subset. The set of ideals of $P$ ordered by set inclusion is denoted by $\operatorname{Idl}(P)$. The poset $\operatorname{Idl}(P)$ becomes a domain called the ideal completion of $P$ if $P$ is countable. We have an order isomorphism $K(\operatorname{Idl}(P)) \cong P$ for each countable poset $P$ with a least element. On the other hand, for a domain $D$, we have $\operatorname{Idl}(K(D)) \cong D$. Therefore, $K(D)$, the set of compact elements of $D$, determines the structure of $D$. We say that an ideal of $K(D)$ is principal if its least upper bound is in $K(D)$. An infinite strictly increasing sequence $d_{0} \subsetneq d_{1} \sqsubseteq d_{2} \sqsubset \cdots$ in $K(D)$ determines a non-principal ideal $\left\{e \in K(D): e \sqsubseteq d_{i}\right.$ for some $\left.i\right\}$ of $K(D)$ and thus determines a point of $L(D)$.

A poset $P$ is a conditional upper semilattice with least element (cusl) if it has a least element and every pair of compatible elements has a least upper bound. If $P$ is a cusl, then $\operatorname{Idl}(P)$ is a bounded complete domain. For background material on domains, see [8, 1, 15]. 
Representation: We write $f: \subseteq A \rightarrow B$ if $f$ is a partial function from $A$ to $B$. For a finite or a countably infinite alphabet $\Sigma$, a surjective partial function from $\Sigma^{\omega}$ to $X$ is called a (TTE-)representation of $X$. We say that a continuous function $\gamma: \subseteq \Sigma^{\omega} \rightarrow X$ is reducible (resp. continuously reducible) to $\delta: \subseteq \Sigma^{\omega} \rightarrow X$ if there exists a computable function (resp. continuous function) $\phi: \subseteq \Sigma^{\omega} \rightarrow \Sigma^{\omega}$ such that $\gamma(p)=\delta(\phi(p))$ for every $p \in \operatorname{dom}(\gamma)$. Two representations $\delta, \delta^{\prime}: \subseteq \Sigma^{\omega} \rightarrow X$ are equivalent (resp. continuously equivalent) if they are reducible (reps. continuously reducible) to each other. A representation $\delta: \subseteq \Sigma^{\omega} \rightarrow X$ is called admissible if $\delta$ is continuous and every continuous function $\gamma: \subseteq \Sigma^{\omega} \rightarrow X$ is continuously reducible to $\delta$.

Let $X$ be a $T_{0}$-space and $B=\left\{B_{n}: n \in \mathbb{N}\right\}$ be a subbase of $X$ indexed by $\mathbb{N}$. Consider the representation $\delta_{B}: \subseteq \mathbb{N}^{\omega} \rightarrow X$ such that $\delta_{B}(p)=x$ if and only if $\{p(k): k \in \mathbb{N}\}=$ $\left\{n \in \mathbb{N}: x \in B_{n}\right\} . \delta_{B}$ is called a standard representation of $X$ with respect to $B$. Any representation which is continuously equivalent to a standard representation is admissible [23, 14, 22].

Domain representation: Let $D$ be a domain, $D^{R}$ a subspace of $D$, and $\mu$ a quotient map from $D^{R}$ onto $X$. The triple $\left(D, D^{R}, \mu\right)$ is called a domain representation of $X$. Note that we do not require $D$ to be bounded-complete or each element of $D^{R}$ to be total (i.e., condense) in this paper. See [2, 3] for the notion of totality. A domain representation is called a retract domain representation if $\mu$ is a retraction, and a homeomorphic domain representation if $\mu$ is a homeomorphism.

A domain representation $\left(D, D^{R}, \mu\right)$ of $X$ is upwards-closed if $D^{R}$ is upwards-closed and $\mu(d)=\mu(e)$ for every $d \sqsupseteq e \in D^{R}$. A domain representation $\left(D, D^{R}, \mu\right)$ is called admissible if for every pair $\left(E, E^{R}\right)$ of a domain $E$ and a dense subset $E^{R} \subseteq E$ and for every continuous function $\nu: E^{R} \rightarrow X$, there is a continuous function $\phi: E \rightarrow D$ such that $\nu(x)=\mu(\phi(x))$ holds for all $x \in E^{R}$. A domain representation $E=\left(E, E^{R}, \nu\right)$ reduces continuously to a domain representation $D=\left(D, D^{R}, \mu\right)$ if there is a continuous function $\phi: E \rightarrow D$ such that $\phi\left(E^{R}\right) \subseteq D^{R}$ and $\nu(x)=\mu(\phi(x))$ for all $x \in E^{R}$. For more about (admissible) domain representations, see [3, 9, 16, 17].

\section{PRoper DYADIC SUBbaseS}

Recall that a space means a Hausdorff space unless otherwise noted.

Definition 2.1. A dyadic subbase $S$ of a space $X$ is a family $\left\{S_{n, a}: n \in \mathbb{N}, a \in \mathbb{2}\right\}$ of regular open sets indexed by $\mathbb{N} \times \mathbb{2}$ such that (1) $S_{n, 1}$ is the exterior of $S_{n, 0}$ for each $n \in \mathbb{N}$ and (2) it forms a subbase of $X$.

Note that we allow duplications in $S_{n, a}$ and therefore, for example, a one point set $X=\{x\}$ has a dyadic subbase $S_{n, 0}=X, S_{n, 1}=\varnothing(n=0,1, \ldots)$. Note also that this definition is applicable also to non-Hausdorff spaces, though we only consider the case $X$ is Hausdorff in this paper. We denote by $S_{n, \perp}$ the set $X \backslash\left(S_{n, 0} \cup S_{n, 1}\right)$. Since $S_{n, 0}$ is regular open, we get bd $S_{n, 0}=\operatorname{bd} S_{n, 1}=S_{n, \perp}$. Note that $S_{n, \perp}$ is defined differently in [20].

A topological space is called semiregular if the family of regular open sets forms a base of $X$. It is immediate that a regular space is semiregular. From the definition, a space with a dyadic subbase is a second-countable semiregular space. On the other hand, it is shown in [20] that every second-countable semiregular space has a dyadic subbase. 


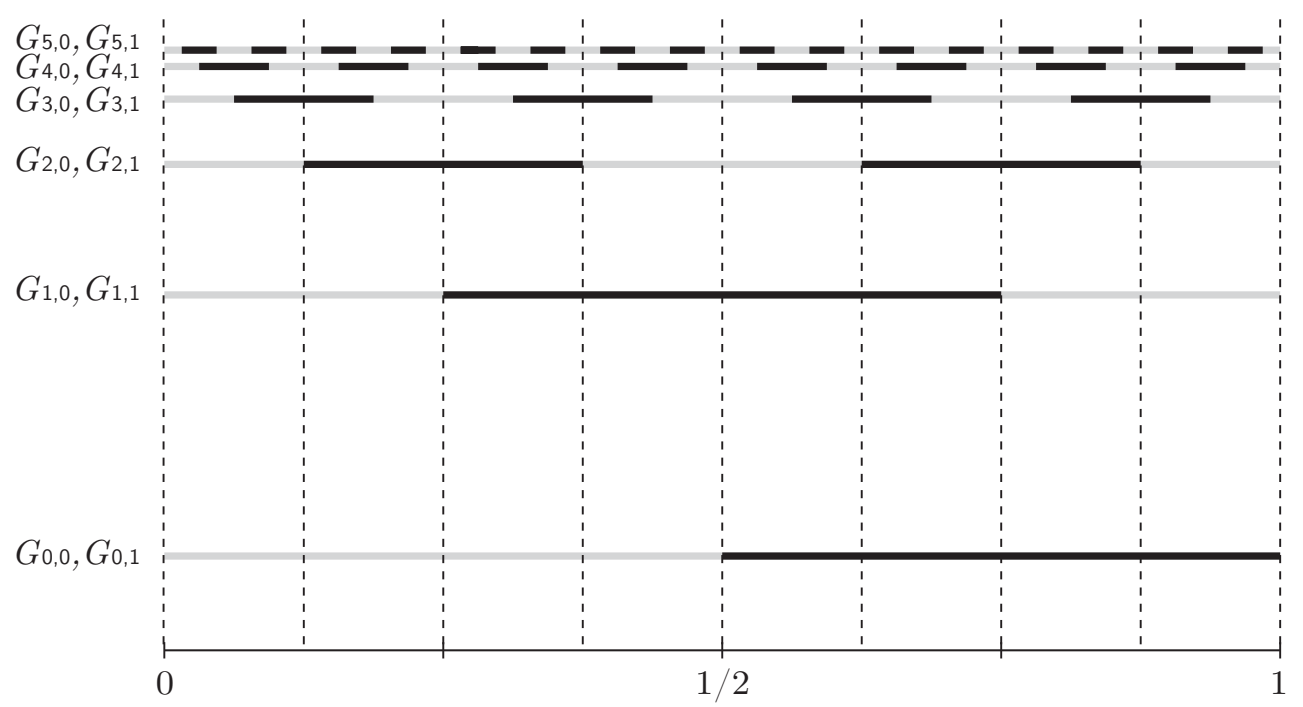

Figure 1: Gray subbase of the unit interval $\mathbb{I}$.

From a dyadic subbase $S$, we obtain a topological embedding $\varphi_{S}: X \rightarrow \mathbb{T}^{\omega}$ as follows.

$$
\varphi_{S}(x)(n)= \begin{cases}0 & \left(x \in S_{n, 0}\right), \\ 1 & \left(x \in S_{n, 1}\right), \\ \perp & \left(x \in S_{n, \perp}\right) .\end{cases}
$$

We denote by $\tilde{x}$ the sequence $\varphi_{S}(x) \in \mathbb{T}^{\omega}$ and denote by $\tilde{X}$ the set $\varphi_{S}(X) \subseteq \mathbb{T}^{\omega}$ if there is no ambiguity of $S$.

In the sequence $\tilde{x}$, if $\tilde{x}(n)=a$ for $n \in \mathbb{N}$ and $a \in \mathcal{Z}$, then this fact holds for some neighbourhood $A$ of $x$ because $S_{n, a}$ is open. On the other hand, if $\tilde{x}(n)=\perp$, then every neighbourhood $A$ of $x$ contains points $y_{0}$ and $y_{1}$ with $\tilde{y}_{0}(n)=0$ and $\tilde{y}_{1}(n)=1$. Therefore, if $\tilde{x}(n)=\perp$, then every neighbourhood $A$ of $x$ does not exclude both of the possibilities $\tilde{x}(n)=0$ and $\tilde{x}(n)=1$.

Example 2.2 (Gray subbase). Let $\mathbb{I}=[0,1]$ be the unit interval and let $X_{0}=[0,1 / 2)$ and $X_{1}=(1 / 2,1]$ be subsets of $\mathbb{I}$. The tent function is the function $t: \mathbb{I} \rightarrow \mathbb{I}$ defined as

$$
t(x)= \begin{cases}2 x & \left(x \in \operatorname{cl} X_{0}\right) \\ 2(1-x) & \left(x \in \operatorname{cl} X_{1}\right)\end{cases}
$$

We define the dyadic subbase $G$ as

$$
G_{n, a}=\left\{x: t^{n}(x) \in X_{a}\right\}
$$

for $n \in \mathbb{N}$ and $a \in \mathcal{Z}$. The map $\varphi_{G}$ is an embedding of the unit interval in $\mathbb{T}^{\omega}$ [7, 18]. If $x$ is a dyadic rational number other than 0 or 1 , then $\varphi_{G}(x)$ has the form $e \perp 10^{\omega}$ for $e \in \mathbb{2}^{*}$, and it is in $\mathbb{2}^{\omega}$ otherwise. Figure 1 shows the Gray subbase, with the gray lines representing $G_{n, 0}$ and the black lines representing $G_{n, 1}$. 
For a dyadic subbase $S$ and $p \in \mathbb{T}^{\omega}$, let

$$
\begin{aligned}
& S(p)=\bigcap_{k \in \operatorname{dom}(p)} S_{k, p(k)}, \\
& \bar{S}(p)=\bigcap_{k \in \operatorname{dom}(p)} \operatorname{cl} S_{k, p(k)}=\bigcap_{k \in \operatorname{dom}(p)}\left(S_{k, p(k)} \cup S_{k, \perp}\right)
\end{aligned}
$$

denote the corresponding subsets of $X$. Note that, for $x \in X$ and $p \in \mathbb{T}^{\omega}$,

$$
\begin{aligned}
& x \in S(p) \Leftrightarrow \tilde{x}(k)=p(k) \text { for } k \in \operatorname{dom}(p) \Leftrightarrow p \sqsubseteq \tilde{x}, \\
& x \in \bar{S}(p) \Leftrightarrow \tilde{x}(k) \sqsubseteq p(k) \text { for } k \in \operatorname{dom}(p) \Leftrightarrow p \uparrow \tilde{x} .
\end{aligned}
$$

For $e \in \mathbb{T}^{*}=K\left(\mathbb{T}^{\omega}\right), S(e)$ is an element of the base generated by the subbase $S$. We denote by $\mathfrak{B}_{S}$ the base $\left\{S(e): e \in \mathbb{T}^{*}\right\} \backslash\{\varnothing\}$. On the other hand, $L\left(\mathbb{T}^{\omega}\right)$ is the space in which $X$ is represented as the following proposition shows.

Proposition 2.3. Suppose that $S$ is a dyadic subbase of a space $X$.

(1) $S(\tilde{x})=\{x\}$ for all $x \in X$.

(2) $\tilde{X} \subseteq L\left(\mathbb{T}^{\omega}\right)$.

Proof.

(1) Let $x, y$ be distinct elements in $X$. Since $X$ is $T_{1}$, there exists $e \in \mathbb{T}^{*}$ such that $x \in S(e)$ and $y \notin S(e)$. From (2.3), we have $e \sqsubseteq \tilde{x}$ and $e \neq \tilde{y}$. So we get $\tilde{x} \nsubseteq \tilde{y}$, therefore, $y \notin S(\tilde{x})$.

(2) Suppose that $\operatorname{dom}(\tilde{x})$ is finite. Then $S(\tilde{x})$ is an open set and thus $\{x\}$ is a clopen set which contradicts the fact that $x$ is on the boundary of $S_{n, a}$ for $n \notin \operatorname{dom}(\tilde{x})$.

Definition 2.4. We say that a dyadic subbase $S$ is proper if $\operatorname{cl} S(e)=\bar{S}(e)$ for every $e \in \mathbb{T}^{*}$.

If $S$ is a proper dyadic subbase, then $\bar{S}(e)$ is the closure of the base element $S(e)$. Therefore, by (2.4), the sequence $\tilde{x}$ codes not only base elements to which $x$ belongs but also base elements to whose closure $x$ belongs.

Proposition 2.5. Suppose that $S$ is a proper dyadic subbase of a space $X$.

(1) If $x \in X$ and $p \sqsupseteq \tilde{x}$, then the family $\left\{S(e): e \in K_{p}\right\}$ is a filter base which converges to $x \in X$.

(2) If $x \neq y \in X$, then $x \in S_{n, a}$ and $y \in S_{n, 1-a}$ for some $n \in \mathbb{N}$ and $a \in \mathbb{2}$. That is, $x$ and $y$ are separated by some subbase element.

(3) If $x \in X$ and $p \sqsupseteq \tilde{x}$, then $\bar{S}(p)=\{x\}$.

(4) If $p \in \mathbb{2}^{\omega}$, then $\bar{S}(p)$ is either a one-point set $\{x\}$ for some $x \in X$ or the empty set.

Proof.

(1) Since we have $\tilde{x} \uparrow e$ for every $e \in K_{p}$, we obtain $\operatorname{cl} S(e)=\bar{S}(e) \neq \varnothing$. Therefore, we get $\varnothing \notin\left\{S(e): e \in K_{p}\right\}$.

(2) Since $X$ is Hausdorff, there is $e \in \mathbb{T}^{*}$ such that $x \in S(e)$ and $y \notin \operatorname{cl} S(e)=\bar{S}(e)$. That is, $e \sqsubseteq \tilde{x}$ and $e \hat{\gamma} \tilde{y}$ by (2.3) and (2.4). Therefore, we get $\tilde{x} \hat{\chi} \tilde{y}$.

(3) From $(2)$, we have $\bar{S}(\tilde{x})=\{x\}$. We get $\bar{S}(p) \subseteq \bar{S}(\tilde{x})=\{x\}$ from $p \sqsupseteq \tilde{x}$, and $\bar{S}(p) \ni x$ from $p \uparrow \tilde{x}$.

(4) Let $p \in \mathbb{2}^{\omega}$. If $p \sqsupseteq \tilde{x}$ for some $x \in X$, then $\bar{S}(p)$ is a one-point set $\{x\}$ by (3). If $p \nsubseteq \tilde{x}$ for all $x \in X$, then $p \hat{\chi} \tilde{x}$, because $p$ is maximal. Therefore, $\bar{S}(p)$ is empty. 
[20] contains an example of a non-proper dyadic subbase for which Proposition 2.5 (1) to (4) do not hold.

Finally, we define a property of a dyadic subbase which is stronger than properness.

Definition 2.6. An independent subbase is a dyadic subbase such that $S(e)$ is not empty for every $e \in \mathbb{T}^{*}$.

Proposition 2.7 ([12]). An independent subbase is proper.

The Gray subbase in Example 2.2 is an independent subbase and we show many independent subbases as examples of proper dyadic subbases. When $S$ is an independent subbase, we have $S(d) \supseteq S(e)$ if and only if $d \sqsubseteq e$. In particular, $S(d) \neq S(e)$ if $d \neq e$. Therefore, for an independent subbase $S$, the poset $\left(\mathfrak{B}_{S}, \supseteq\right)$ ordered by reverse inclusion is isomorphic to $\mathbb{T}^{*}$.

\section{REPRESENTATIONS AND DOMAIN REPRESENTATIONS DERIVED FROM DYADIC SUBBASES}

We study some representations and domain representations of a space $X$ derived from a (proper) dyadic subbase of $X$.

We introduce two representations. The first one is immediately derived from a dyadic subbase. If $S$ is a dyadic subbase of $X$, then the inverse $\varphi_{S}^{-1}$ of the embedding $\varphi_{S}$ is a representation of $X$ with the alphabet $\Gamma=\{0,1, \perp\}$ where $\perp$ is considered as an ordinary character of $\Gamma$. Each point is represented uniquely with this representation and it is easy to show that $\varphi_{S}^{-1}: \subseteq \Gamma^{\omega} \rightarrow X$ is an admissible representation if and only if $S_{n, \perp}=\varnothing$ for every $n$.

The second one is derived from a proper dyadic subbase. If $S$ is a proper dyadic subbase of $X$, from Proposition 2.5 (3), we have a map $\rho_{S}$ from $\uparrow \tilde{X} \subset \mathbb{T}^{\omega}$ to $X$ such that $\rho_{S}(p)$ is the unique element in $\bar{S}(p)$. In particular, from Proposition 2.5 (4), $\rho_{S}$ restricted to the maximal elements $\mathcal{2}^{\omega}$ is a partial surjective map from $\mathcal{2}^{\omega}$ to $X$, that is, it is a representation of $X$ which we denote by $\rho_{S}^{\prime}$.

Example 3.1. For the Gray subbase $G$ of $\mathbb{I}, \rho_{G}^{\prime}$ is a total function from $\mathcal{2}^{\omega}$ to $\mathbb{I}$ which is called the Gray expansion of $\mathbb{I}[18] . \rho_{G}^{\prime}$ is equivalent to the binary expansion through simple conversion functions.

As this example suggests, we consider that $\rho_{S}^{\prime}$ is a generalization of the binary expansion representation to a proper dyadic subbase $S$. We study its continuity in Proposition 3.4. It is not admissible in general as the following proposition shows.

Proposition 3.2. Suppose that $S$ is a proper dyadic subbase of a space $X$. $\rho_{S}^{\prime}$ is admissible if and only if $S_{n, \perp}=\varnothing$ for every $n$.

Proof. Only if part: suppose that $\rho_{S}^{\prime}$ is admissible and $x \in S_{n, \perp}$. Theorem 12 of [4] says that every admissible representation has a continuously equivalent open restriction. Suppose that $\delta: \subseteq \mathbb{2}^{\omega} \rightarrow X$ is such an open restriction of $\rho_{S}^{\prime}$ and $x=\delta(p)$. Let $a=p(n)$. Since $\delta$ is an open map, $\delta\left(\left\{q \in \mathbb{2}^{\omega}: q(n)=a\right\}\right)$ is an open neighbourhood of $x$, and since $\delta$ is a restriction of $\rho_{S}^{\prime}, \delta\left(\left\{q \in \mathbb{2}^{\omega}: q(n)=a\right\}\right) \subseteq \rho_{S}^{\prime}\left(\left\{q \in \mathbb{P}^{\omega}: q(n)=a\right\}\right)=S_{n, a} \cup S_{n, \perp}$. Therefore, $S_{n, a} \cup S_{n, \perp}$ is a neighbourhood of $x$, which contradicts with $x \in S_{n, \perp}$.

If part: since the base $\mathfrak{B}_{S}$ is composed of closed and open sets, $X$ is regular and therefore $\rho_{S}^{\prime}$ is continuous by Proposition 3.4 below. Since $S_{n, \perp}$ is empty, $x \in S_{n, 0}$ or $x \in S_{n, 1}$ holds 
for every $x \in X$. Therefore, one can construct a reduction from the standard representation of $X$ with respect to an enumeration of the subbase $S$ to $\rho_{S}^{\prime}$.

Next, we study domain representations. We start with a general construction of a domain representation from a base of a space. Suppose that $\mathfrak{B}$ is a base of a space $X$ such that $\varnothing \notin \mathfrak{B}, X \in \mathfrak{B}$, and $\mathfrak{B}$ is closed under finite non-empty intersection. For the domain $D_{\mathfrak{B}}$ obtained as the ideal completion of the poset $(\mathfrak{B}, \supseteq)$ with the reverse inclusion and for the map $\iota(x)=\{U \in \mathfrak{B}: x \in U\}$ from $X$ to $D_{\mathfrak{B}}, \iota$ is a homeomorphic embedding of $X$ in $D_{\mathfrak{B}}$. Therefore, $\left(D_{\mathfrak{B}}, \iota(X), \iota^{-1}\right)$ is a homeomorphic domain representation which is known to be admissible [3, 9, 17].

We introduce two domain representations derived from (proper) dyadic subbases. The first one is $\left(\mathbb{T}^{\omega}, \tilde{X}, \varphi_{S}^{-1}\right)$, which is defined for a space $X$ with a dyadic subbase $S$. Since $\varphi_{S}$ is an embedding, it is a homeomorphic domain representation. In particular, if $S$ is an independent subbase, then the poset $\mathfrak{B}_{S}$ is isomorphic to the poset $\mathbb{T}^{*}$. Therefore, the domain $D_{\mathfrak{B}_{S}}$ is isomorphic to $\mathbb{T}^{\omega}$ and thus the domain representations $\left(\mathbb{T}^{\omega}, \tilde{X}, \varphi_{S}^{-1}\right)$ and $\left(D_{\mathfrak{B}_{S}}, \iota(X), \iota^{-1}\right)$ coincide. However, if $S$ is a dyadic subbase which is not independent, then the poset $\mathbb{T}^{*}$ provides only a "notation" of the base $\mathfrak{B}_{S}$, and a set $S(d)$ may be the same as $S(e)$ for $d \neq e \in \mathbb{T}^{*}$. We show that $\left(\mathbb{T}^{\omega}, \tilde{X}, \varphi_{S}^{-1}\right)$ is an admissible domain representation even for this case.

Proposition 3.3. If $S$ is a dyadic subbase of a space $X$, then $\left(\mathbb{T}^{\omega}, \tilde{X}, \varphi_{S}^{-1}\right)$ is an admissible domain representation.

Proof. Suppose that $E^{R}$ is a subset of a domain $E$ and $\mu$ is a continuous map from $E^{R}$ to $X$. Define a function $\phi: K(E) \rightarrow \mathbb{T}^{\omega}$ as $\phi(e)(n)=a$ if and only if $\mu\left(\uparrow e \cap E^{R}\right) \subseteq S_{n, a}$. Since $\phi$ is monotonic, it has a continuous extension to $E$, which is a continuous function from $E$ to $\mathbb{T}^{\omega}$. It is also denoted by $\phi$. We show that the function $\phi$ satisfies $\varphi_{S}^{-1}(\phi(p))=\mu(p)$ for $p \in E^{R}$. We have $\phi(p)(n)=\sqcup_{e \in K_{p}} \phi(e)(n)$. Therefore, for $a \in \mathcal{Z}, \phi(p)(n)=a$ if and only if $\left(\exists e \in K_{p}\right)(\phi(e)(n)=a)$, if and only if $\left(\exists e \in K_{p}\right)\left(\mu\left(\uparrow e \cap E^{R}\right) \subseteq S_{n, a}\right)$, if and only if $\mu(p) \in S_{n, a}$. Therefore, $\phi(p)=\varphi_{S}(\mu(p))$.

The other domain representation is $\left(\mathbb{T}^{\omega}, \uparrow \tilde{X}, \rho_{S}\right)$, which is defined for a regular space $X$ with a proper dyadic subbase $S$. Suppose that $S$ is a proper dyadic subbase of a space $X$. From Proposition 2.5, $\rho_{S}$ is a map from $\uparrow \widetilde{X}$ to $X$. We have $\varphi_{S}\left(\rho_{S}(p)\right) \sqsubseteq p$ and $\rho_{S}\left(\varphi_{S}(x)\right)=$ $x$. Therefore, $\left(\mathbb{T}^{\omega}, \uparrow \tilde{X}, \rho_{S}\right)$ is an upwards-closed retract domain representation if and only if $\rho_{S}$ is a quotient map. Blanck showed in Theorem 5.10 of [3] that if a topological space has an upwards-closed retract Scott domain representation, then it is a regular Hausdorff space. Therefore, $\left(\mathbb{T}^{\omega}, \uparrow \tilde{X}, \rho_{S}\right)$ is a domain representation only if $X$ is regular. We show this fact as a corollary to the following equivalence.

Proposition 3.4. Let $S$ be a proper dyadic subbase of a space $X$. The followings are equivalent.

(1) $X$ is regular.

(2) $\rho_{S}: \uparrow \tilde{X} \rightarrow X$ is continuous.

(3) $\rho_{S}^{\prime}: \subseteq \mathbb{2}^{\omega} \rightarrow X$ is continuous.

Proof.

$(1 \Rightarrow 2)$ : Let $p \in \uparrow \tilde{X}$ and $x=\rho_{S}(p)$. Since $\left\{S\left(\left.\tilde{x}\right|_{n}\right): n \in \mathbb{N}\right\}$ is a neighbourhood base of $x$ in $X$ and $\left\{\left.\uparrow p\right|_{m} \cap \uparrow \widetilde{X}: m \in \mathbb{N}\right\}$ is a neighbourhood base of $p$ in $\uparrow \widetilde{X}$, it suffices to show 
that for every $n$, there is $m$ such that $\rho_{S}\left(\left.\uparrow p\right|_{m} \cap \uparrow \tilde{X}\right) \subseteq S\left(\left.\tilde{x}\right|_{n}\right)$. Since $X$ is regular, there is $m>n$ such that $x \in S\left(\left.\tilde{x}\right|_{m}\right) \subseteq \operatorname{cl} S\left(\left.\tilde{x}\right|_{m}\right) \subseteq S\left(\left.\tilde{x}\right|_{n}\right)$. Then, for all $q \in \uparrow \tilde{X}$ such that $\left.q \sqsupseteq p\right|_{m}$, we have $\left.\varphi_{S}\left(\rho_{S}(q)\right) \uparrow \tilde{x}\right|_{m}$ because $\left.\left.\varphi_{S}\left(\rho_{S}(q)\right) \sqsubseteq q \sqsupseteq p\right|_{m} \sqsupseteq \tilde{x}\right|_{m}$. Thus, $\rho_{S}(q) \in \bar{S}\left(\left.\tilde{x}\right|_{m}\right)$. Therefore, $\rho_{S}(q) \in \bar{S}\left(\left.\tilde{x}\right|_{m}\right)=\operatorname{cl} S\left(\left.\tilde{x}\right|_{m}\right) \subseteq S\left(\left.\tilde{x}\right|_{n}\right)$.

$(2 \Rightarrow 3)$ : Immediate.

$(3 \Rightarrow 1)$ : Suppose that $x \in X$ and $n \in \mathbb{N}$. For each $p \in \uparrow \tilde{x} \cap \mathbb{2}^{\omega}$, since $\rho_{S}^{\prime}$ is continuous on $p$, there exists $e_{p} \in K_{p}$ such that $\rho_{S}^{\prime}\left(\uparrow e_{p} \cap \mathbb{2}^{\omega}\right) \subseteq S\left(\left.\tilde{x}\right|_{n}\right)$. It means that $\bar{S}\left(e_{p}\right) \subseteq S\left(\left.\tilde{x}\right|_{n}\right)$. Here, we can assume that $\left.\tilde{x}\right|_{\left|e_{p}\right|} \sqsubseteq e_{p}$ by replacing $e_{p}$ with $\left.e_{p} \sqcup \tilde{x}\right|_{\left|e_{p}\right|}$. Note that $\uparrow e_{p} \cap \mathbb{Q}^{\omega}$ for $p \in \uparrow \tilde{x} \cap \mathbb{2}^{\omega}$ is an open cover of $\uparrow \tilde{x} \cap \mathbb{2}^{\omega}$ and $\uparrow \tilde{x} \cap \mathcal{2}^{\omega}$ is compact because it is homeomorphic to $\mathbb{2}^{j}$ for some $j \leqslant \omega$. Therefore, for some finite subset $\left\{p_{0}, \ldots, p_{h-1}\right\}$ of $\uparrow \tilde{x} \cap \mathbb{2}^{\omega}$, we have $\cup_{i<h} \uparrow e_{p_{i}} \supseteq \uparrow \tilde{x} \cap \mathbb{2}^{\omega}$. Let $m$ be the maximal length of $e_{p_{i}}$ for $i<h$ and let $l=m-\left|\operatorname{dom}\left(\left.x\right|_{m}\right)\right|$. Let $d_{0}, \ldots, d_{2^{l}-1}$ be sequences of length $m$ obtained by filling the first $l$ bottoms of $\left.\tilde{x}\right|_{m}$ with 0 and 1 . We have $\left(\uparrow d_{0} \cup \ldots \cup \uparrow d_{2^{l}-1}\right) \cap \mathbb{2}^{\omega}=\left.\uparrow \tilde{x}\right|_{m} \cap \mathbb{2}^{\omega}$. Therefore, $\cup_{i<2^{l}-1} \bar{S}\left(d_{i}\right)=\bar{S}\left(\left.\tilde{x}\right|_{m}\right)$. On the other hand, for each $i<2^{l}$, there is $j<h$ such that $d_{i} \sqsupseteq e_{p_{j}}$. Therefore, $\bar{S}\left(d_{i}\right) \subseteq \bar{S}\left(e_{p_{j}}\right) \subseteq S\left(\left.\tilde{x}\right|_{n}\right)$. Thus, we have $\bar{S}\left(\left.\tilde{x}\right|_{m}\right) \subseteq S\left(\left.\tilde{x}\right|_{n}\right)$. Since $\bar{S}\left(\left.\tilde{x}\right|_{m}\right)=\operatorname{cl} S\left(\left.\tilde{x}\right|_{m}\right)$, it means that $X$ is a regular space.

Corollary 3.5. Suppose that $S$ is a proper dyadic subbase of a space $X$. The triple $\left(\mathbb{T}^{\omega}, \uparrow \tilde{X}, \rho_{S}\right)$ is a domain representation if and only if $X$ is regular. In this case, it is an admissible retract domain representation.

Proof. Suppose that $X$ is regular. From Proposition 3.4, $\rho_{S}$ is a retraction with right inverse $\varphi_{S}$. Therefore, $\rho_{S}$ is a quotient map. Since $\tilde{X} \subseteq \uparrow \widetilde{X}$ and $\varphi_{S}^{-1}$ is a restriction of $\rho_{S}$ to $\tilde{X}$, the identity map on $\mathbb{T}^{\omega}$ is a reduction map from the admissible domain representation $\left(\mathbb{T}^{\omega}, \tilde{X}, \varphi_{S}^{-1}\right)$ to $\left(\mathbb{T}^{\omega}, \uparrow \tilde{X}, \rho_{S}\right)$.

\section{Domains $D_{S}$ And $\widehat{D}_{S}$}

In the previous section, we studied domain representations in the domain $\mathbb{T}^{\omega}$. In the following sections, we study domain representations in subdomains $D_{S}$ and $\widehat{D}_{S}$ of $\mathbb{T}^{\omega}$. Before that, we consider the domain $E_{S}$ which is defined as the closure of $\widetilde{X}$ in $\mathbb{T}^{\omega}$. It is easy to show that the triple $\left(E_{S}, \tilde{X}, \varphi_{S}^{-1}\right)$ is a dense domain representation of $X$ and, if in addition $S$ is proper and $X$ is regular, then $\left(E_{S}, \uparrow \tilde{X}, \rho_{S}\right)$ is a dense admissible retract domain representation of $X$. In these domain representations, we have $S(e) \neq \varnothing$ for every $e \in K\left(E_{S}\right)$ and the family $\left\{S(e): e \in K_{p}\right\}$ forms a filter base for every $p \in L\left(E_{S}\right)$. In this sense, one can say that $E_{S}$ does not contain superfluous elements. However, $E_{S}$ is identical to $\mathbb{T}^{\omega}$ if $S$ is an independent subbase and the domain $E_{S}$ does not have information about $X$ in this case. We consider further restrictions of $\mathbb{T}^{\omega}$ and define the domains $D_{S}$ and $\widehat{D}_{S}$ as follows.

Definition 4.1. Let $S$ be a dyadic subbase of a space $X$.

(1) We define the poset $K_{S} \subseteq \mathbb{T}^{*}$ as

$$
K_{S}=\left\{\left.p\right|_{m}: p \in \tilde{X}, m \in \mathbb{N}\right\}
$$

and define $D_{S}=\operatorname{Idl}\left(K_{S}\right)$. 

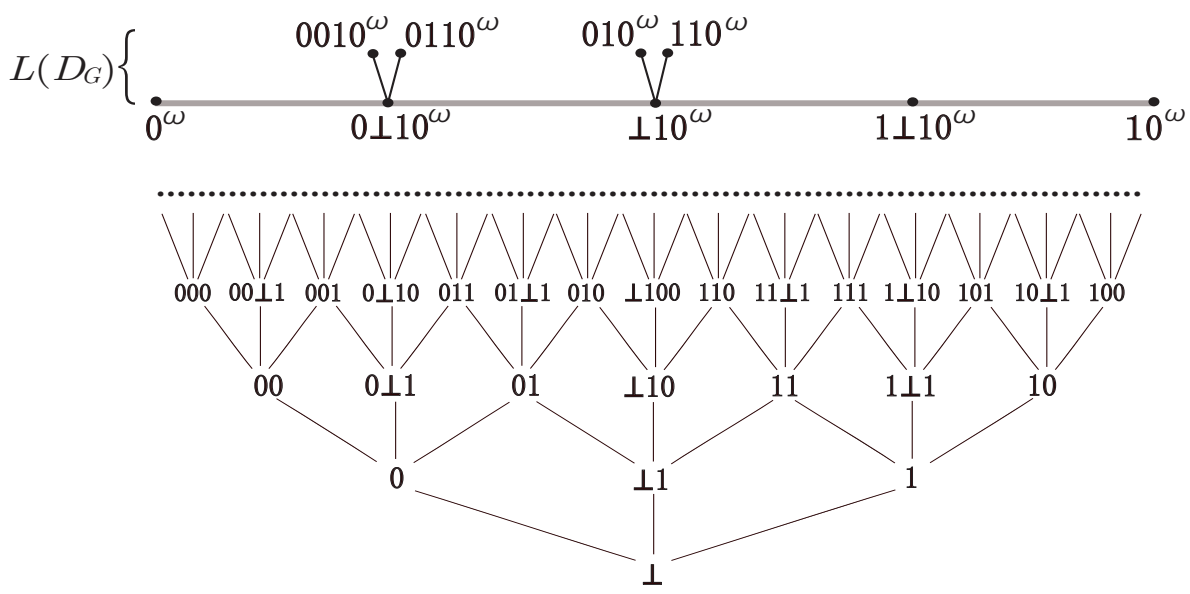

Figure 2: The domain $D_{G}$.

(2) We define the poset $\widehat{K}_{S} \subseteq \mathbb{T}^{*}$ as

$$
\widehat{K}_{S}=\left\{\left.p\right|_{m}: p \in \uparrow \tilde{X}, m \in \mathbb{N}\right\}
$$

and define $\widehat{D}_{S}=\operatorname{Idl}\left(\widehat{K}_{S}\right)$.

For the Gray-subbase $G$ of $\mathbb{I}$, we have $\perp 1 \in K_{G}$ because $\varphi_{G}(1 / 2)=\perp 10^{\omega}$, but $\perp 0 \notin K_{G}$ and $\perp \perp 1 \notin K_{G}$. Figure 2 shows the structure of $D_{G}=\widehat{D}_{G}$.

We have $K_{S} \subseteq \widehat{K}_{S} \subseteq \mathbb{T}^{*}$ and $D_{S} \subseteq \widehat{D}_{S} \subseteq \mathbb{T}^{\omega}$ for a dyadic subbase $S$. We also have $\tilde{X} \subseteq D_{S}$ and $\uparrow \tilde{X} \subseteq \widehat{D}_{S}$.

\section{Proposition 4.2.}

(1) If $S$ is a dyadic subbase of a space $X$, then $\tilde{X}$ is dense in $D_{S}$.

(2) If $S$ is a proper dyadic subbase of a space $X$, then $\tilde{X}$ is dense in $\widehat{D}_{S}$.

Proof.

(1) $\tilde{X}$ is dense in $D_{S}$ because $S(e)$ is not empty for every $e \in K_{S}$.

(2) By Proposition [2.5(1), $S(e)$ is not empty for every $e \in \widehat{K}_{S}$.

The domains $D_{S}$ and $\widehat{D}_{S}$ are not equal in general as the following example shows.

Example 4.3. Let $W$ be the space obtained by glueing four copies of $\mathbb{I}$ at one of the endpoints. That is, $W=2 \times 2 \times \mathbb{I} / \sim$ for $\sim$ the equivalence relation identifying $(a, b, 0)$ for $a, b \in \mathcal{2}$. Let $z$ be the identified point. That is, $z=[(a, b, 0)]$ for $a, b \in \mathcal{2}$. Let $R$ be the dyadic subbase defined as

$$
\begin{aligned}
R_{0, c} & =\{c\} \times \mathcal{2} \times(0,1] / \sim, \\
R_{1, c} & =\mathcal{2} \times\{c\} \times(0,1] / \sim, \\
R_{n+2, c} & =\mathcal{2} \times \mathcal{2} \times G_{n, c} / \sim,
\end{aligned}
$$

for $n \in \mathbb{N}$ and $c \in \mathcal{Z}$. We have $\tilde{z}=\perp \perp 0^{\omega} \in L\left(D_{R}\right)$ and $a b 0^{\omega} \in L\left(D_{R}\right)$ for $a, b \in \mathcal{2}$. However, $a \perp 0^{\omega} \notin L\left(D_{R}\right)$ for $a \in \mathbb{Z}$ and $\perp b 0^{\omega} \notin L\left(D_{R}\right)$ for $b \in \mathcal{Z}$. On the other hand, we have $a \perp 0^{\omega} \in L\left(\widehat{D}_{R}\right)$ for $a \in \mathcal{Z}$ and $\perp b 0^{\omega} \in L\left(\widehat{D}_{R}\right)$ for $b \in \mathcal{L}$. 
Proposition 4.4. If $S$ is a dyadic subbase of a space $X$, then $\widehat{K}_{S}$ is a cusl and therefore $\widehat{D}_{S}$ is a bounded complete domain.

Proof. Let $d=\left.p\right|_{m}, e=\left.q\right|_{n} \in \widehat{K}_{S}$ for $p, q \in \uparrow \tilde{X}$ and $m \leqslant n \in \mathbb{N}$. Suppose that $d \uparrow e$ in $\widehat{K}_{S}$ and let $f=d \sqcup e$ be their least upper bound in $\mathbb{T}^{*}$. Then, since $d$ and $\left.q\right|_{n}$ are compatible and $|d| \leqslant n, d \uparrow q$ in $\mathbb{T}^{\omega}$ and $r=d \sqcup q$ satisfies $\left.r\right|_{n}=f$. Since $r \sqsupseteq q \sqsupseteq \tilde{x}$ for some $x \in X$, we have $f \in \widehat{K}_{S}$.

\section{Proposition 4.5.}

- If $S$ is a dyadic subbase of a space $X$, then the domain representation $\left(\widehat{D}_{S}, \tilde{X}, \varphi_{S}^{-1}\right)$ is admissible.

- If $S$ is a proper dyadic subbase of a regular space $X$, then the domain representation $\left(\widehat{D}_{S}, \uparrow \tilde{X}, \rho_{S}\right)$ is admissible.

Proof.

(1) We show that there is a reduction from the admissible domain representation $\left(\mathbb{T}^{\omega}, \tilde{X}, \varphi_{S}^{-1}\right)$ to $\left(\widehat{D}_{S}, \tilde{X}, \varphi_{S}^{-1}\right)$. Since $\widehat{D}_{S}$ is bounded complete, we define $\phi: \mathbb{T}^{\omega} \rightarrow \widehat{D}_{S}$ as $\phi(p)=\sqcup\{e \in$ $\left.\widehat{K}_{S}: e \sqsubseteq p\right\}$. It preserves $\widetilde{X}$ because $\left\{S(e): e \in \widehat{K}_{S}, e \sqsubseteq \tilde{x}\right\}$ contains $S\left(\left.\tilde{x}\right|_{n}\right)$ for every $n$.

(2) The map $\phi$ preserves $\uparrow \tilde{X}$ and it is a reduction also from $\left(\mathbb{T}^{\omega}, \uparrow \tilde{X}, \rho_{S}\right)$ to $\left(\widehat{D}_{S}, \uparrow \tilde{X}, \rho_{S}\right)$.

As Example 4.6 shows, $D_{S}$ is not bounded complete in general. It is left open whether the results corresponding to Proposition 4.5 hold for $D_{S}$.

Example 4.6. Let $Y$ be the space obtained by glueing $1 / 4$ and $3 / 4$ in $\mathbb{I}$. That is, $Y$ is the quotient space of $\mathbb{I}$ with the equivalence relation generated by $1 / 4 \sim 3 / 4$. Let $T$ be the independent subbase of $Y$ such that $T_{0,0}=\left(G_{0,0} \backslash\{1 / 4\}\right) / \sim, T_{0,1}=\left(G_{0,1} \backslash\{3 / 4\}\right) / \sim$, and $T_{n, a}=G_{n, a} / \sim$ for $n>0$. We have $\varphi_{T}(z)=\perp \perp 10^{\omega}$ for $z=[1 / 4]=[3 / 4]$ and $\varphi_{T}([x])=\varphi_{G}(x)$ for $x \notin\{1 / 4,3 / 4\}$. Therefore, $K_{S}$ contains $\perp \perp 1$ and $\perp 1\left(=\left.\varphi_{T}([1 / 2])\right|_{2}\right)$, which are bounded above by $011=\left.\varphi_{T}([1 / 3])\right|_{3}$ and $111=\left.\varphi_{T}([2 / 3])\right|_{3}$. However, $\perp 11$, which is the least upper bound of $\perp \perp 1$ and $\perp 1$ in $\mathbb{T}^{\omega}$, does not belong to $K_{T}$. Therefore, $K_{T}$ is not a cusl and $D_{T}$ is not a bounded complete domain. Note that the poset $\widehat{K}_{T}$ contains $\perp 11$ because $\perp 110^{\omega} \sqsupseteq \varphi_{T}(z)$.

In Example 4.6, $\uparrow \varphi_{T}(z)$ in $D_{T}$ is the set $\{\perp \perp p, 00 p, 01 p, 10 p, 11 p, 1 \perp p, 0 \perp p\}$ for $p=10^{\omega}$. Therefore, it is different from $\uparrow \varphi_{T}(z)$ in $\mathbb{T}^{\omega}$ which contains also $\perp 0 p$ and $\perp 1 p$. As Example 4.6 and 4.3 show, $L\left(D_{S}\right) \subsetneq L\left(\hat{D}_{S}\right)$ in general. However, for a proper dyadic subbase $S, D_{S}$ and $\widehat{D}_{S}$ coincide on the top elements as Proposition 4.8 shows.

Lemma 4.7. Let $S$ be a dyadic subbase of a space $X$.

(1) For $p \in D_{S}$ and $n \in \mathbb{N}$, we have $\left.p\right|_{n} \in K_{S}$.

(2) For $p \in \widehat{D}_{S}$ and $n \in \mathbb{N}$, we have $\left.p\right|_{n} \in \widehat{K}_{S}$.

Proof.

(1) Suppose that $p$ is the least upper bound of an ideal $\left\{\left.\tilde{x}_{i}\right|_{m_{i}}: i \in I\right\}$. Then, $\left.p\right|_{n}$ is the least upper bound of the ideal $\left\{\left.\left.\tilde{x}_{i}\right|_{m_{i}}\right|_{n}: i \in I\right\}=\left\{\left.\tilde{x}_{i}\right|_{\min \left\{m_{i}, n\right\}}: i \in I\right\}$, whose length is no more than $n$.

(2) It is proved similarly to (1). 
Proposition 4.8. For a proper dyadic subbase $S$ of a space $X, D_{S} \cap \mathbb{2}^{\omega}=\widehat{D}_{S} \cap \mathcal{2}^{\omega}$.

Proof. Let $p \in \widehat{D}_{S} \cap \mathcal{Q}^{\omega}$ and $n \in \mathbb{N}$. By Lemma 4.7, we have $\left.p\right|_{n} \in \widehat{K}_{S}$. Therefore, $S\left(\left.p\right|_{n}\right) \neq \varnothing$ by Proposition $4.2(2)$. For every $y \in S\left(\left.p\right|_{n}\right)$, we have $\left.p\right|_{n}=\left.\tilde{y}\right|_{n}$ since $p \in \mathbb{2}^{\omega}$. Thus, $\left.p\right|_{n} \in K_{S}$ for every $n \in \mathbb{N}$ and we have $p \in D_{S}$.

\section{Domains With Minimal-Limit Sets}

We study structures of $D_{S}$ and $\widehat{D}_{S}$ and present a condition on $X$ which ensures the existence of minimal elements of $L\left(D_{S}\right)$ and $L\left(\hat{D}_{S}\right)$.

Definition 5.1. Let $P$ be a poset.

(1) $x \in P$ is a minimal element if $y \sqsubseteq x$ implies $y=x$ for all $y \in P$. We write $\min (P)$ for the set of all minimal elements of $P$.

(2) We say that $P$ has enough minimal elements if, for all $y \in P$, there exists $x \in \min (P)$ such that $x \sqsubseteq y$.

(3) For a domain $D$, if $L(D)$ has enough minimal elements, we call $\min (L(D))$ the minimallimit set of $D$.

The poset $L\left(\mathbb{T}^{\omega}\right)$ does not have enough minimal elements.

\section{Definition 5.2.}

(1) Let $(P, \sqsubseteq)$ be a pointed poset with the least element $\perp_{P}$. The level of $x \in P$, if it exists, is the maximal length $n$ of a chain $\perp_{P}=y_{0} \sqsubset y_{1} \subsetneq \ldots \sqsubset y_{n}=x$, and it is denoted by level $(x)$.

(2) A poset $P$ is stratified if it is pointed and every element of $P$ has a level.

(3) We say that $y$ is an immediate successor of $x$ if $x \sqsubset y$ and there is no element $z$ such that $x \sqsubset z \sqsubset y$. We write $\operatorname{succ}(x)$ for the set of immediate successors of $x$.

(4) We say that a stratified poset $P$ is finite-branching if $\operatorname{succ}(x)$ is finite for every $x \in P$.

In [19], the following proposition is proved with a slightly stronger definition of finitebranchingness that contains the condition level $(y)=\operatorname{level}(x)+1$ for $y \in \operatorname{succ}(x)$. However, one can check that this condition is not used in the proof and it holds with our definition of finite-branchingness.

Proposition 5.3 (Proposition 4.13 of [19]). If $D$ is a domain such that $K(D)$ is finitebranching, then $L(D)$ has enough minimal elements and $\min (L(D))$ is compact.

\section{Definition 5.4.}

(1) We say that a space $X$ is adhesive if $X$ has at least two points and closures of any two non-empty open sets have non-empty intersection.

(2) We say that $X$ is nonadhesive if it is not adhesive.

(3) We say that $X$ is strongly nonadhesive if every open subspace is nonadhesive.

Nonadhesiveness (and even strongly nonadhesiveness) is a weak condition that many of the Hausdorff spaces satisfy. A space is called Urysohn (or completely Hausdorff or $T_{2 \frac{1}{2}}$ in some literature) if any two distinct points can be separated by closed neighbourhoods. A regular space is always Urysohn.

Proposition 5.5. Every Urysohn space is strongly nonadhesive. 
Note that there is an adhesive Hausdorff space as the following example shows.

Example 5.6. Let $P$ be the set of dyadic irrational numbers in $\mathbb{I}=[0,1]$ and $\mathbb{N}^{+}$be the set of positive integers. We define our space $A=P \cup \mathbb{N}^{+}$. A neighbourhood base of $x \in P$ is $U \cap P$ for $U$ a Euclidean neighbourhood of $x \in \mathbb{I}$. A neighbourhood base of $n \in \mathbb{N}^{+}$is the union of $\{n\}$ and $U \cap P$ for $U$ a Euclidean neighbourhood of $\left\{k / 2^{n}: k\right.$ is an odd number, $0<$ $\left.k<2^{n}\right\}$. One can easily verify that $A$ is Hausdorff. The closure of $U \cap P$ is $(U \cap P) \cup\{n \in$ $\mathbb{N}^{+}: k / 2^{n} \in U$ for some odd number $\left.k\right\}$ and it contains $\left\{n \in \mathbb{N}^{+}: n>m\right\}$ for some $m$. Therefore $A$ is adhesive. The space $A$ has the following independent subbase $S^{A}$.

$$
S_{n, a}^{A}=\left(G_{n, a} \cap P\right) \cup\left\{n \in \mathbb{N}^{+}: k / 2^{n} \in G_{n, a} \text { for all odd number } k<2^{n}\right\} .
$$

We have $\varphi_{S^{A}}(x)=\varphi_{G}(x)$ for $x \in P$, and $\varphi_{S^{A}}(n)=\perp^{n} 10^{\omega}$ for $n \in \mathbb{N}^{+}$.

As Propositions 5.7 and 5.8 show, adhesiveness of $X$ and finite-branchingness of $D_{S}$ are closely related. Recall again that a (non)adhesive space means a (non)adhesive Hausdorff space.

Proposition 5.7. Suppose that $X$ is an adhesive space and $S$ is a proper dyadic subbase of $X$ such that $S_{n, a} \neq \varnothing$ for every $n \in \mathbb{N}$ and $a \in \mathcal{2}$. Then, $\operatorname{succ}\left(\perp^{\omega}\right)$ in $K_{S}$ is infinite. Therefore, $K_{S}$ is not finite-branching.

Proof. All the elements of $\operatorname{succ}\left(\perp^{\omega}\right)$ have the form $\perp^{k} a$ for $k \in \mathbb{N}$ and $a \in \mathcal{2}$. Suppose that $\operatorname{succ}\left(\perp^{\omega}\right)$ is finite and let $\perp^{n-1} a$ be an element with the maximal length. For $b=1-a$, take $x \in S_{n, b}$ and $p \in \uparrow \tilde{x} \cap \mathcal{2}^{\omega}$. For $d=\left.p\right|_{n}, d \in \widehat{K}_{S}$ holds and therefore $S(d) \neq \varnothing$ by Proposition 4.2. Let $e \in \mathbb{2}^{n}$ be the bitwise complement of $d$. Since $\perp^{n-1} a \in K_{S}, e \in \widehat{K}_{S}$ and therefore $S(e) \neq \varnothing$ by Proposition 4.2. Therefore, closures of $S(d)$ and $S(e)$ intersect. Since $S$ is proper, $\operatorname{cl} S(d)=\bar{S}(d)$ and $\operatorname{cl} S(e)=\bar{S}(e)$. Therefore, there exists $y \in \bar{S}(d) \cap \bar{S}(e)$. Since $\left.\tilde{y}\right|_{n}=\perp^{\omega}$, the smallest index of digits in $\tilde{y}$ is greater than $n$, and we have contradiction.

For the independent subbase $S^{A}$ of $A$ in Example [5.6, $\operatorname{succ}\left(\perp^{\omega}\right)=\left\{\perp^{k} 1: k \in \mathbb{N}\right\}$.

Proposition 5.8. Suppose that $X$ is a nonadhesive space and $S$ is a proper dyadic subbase of $X$. Then, $\operatorname{succ}\left(\perp^{\omega}\right)$ in $K_{S}$ is finite.

Proof. Since $S$ is nonadhesive, for some $p, q \in \mathbb{T}^{*}, S(p) \neq \varnothing, S(q) \neq \varnothing$, and $\operatorname{cl} S(p) \cap$ $\operatorname{cl} S(q)=\varnothing$ hold. Since $S$ is proper, $\bar{S}(p) \cap \bar{S}(q)=\varnothing$. Let $n=\max \{|p|,|q|\}$. If $\left.\tilde{x}\right|_{n}=\perp^{\omega}$ for some $x \in X$, then $x \in \bar{S}(p)$ and $x \in \bar{S}(q)$ and we have contradiction. Thus, in $K_{S}$, $\operatorname{succ}\left(\perp^{\omega}\right) \subseteq\left\{\perp^{k} a \perp^{\omega}: k<n, a \in \mathbb{2}\right\}$.

Lemma 5.9. Suppose that $S$ is a proper dyadic subbase of a space $X$ and $e \in \mathbb{T}^{*}$. Let $\nu$ be an enumeration of $\mathbb{N} \backslash \operatorname{dom}(e)$. Then,

$$
T_{n, a}=S_{\nu(n), a} \cap S(e) \quad(n \in \mathbb{N}, a \in \mathcal{Z})
$$

is a proper dyadic subbase of $S(e)$.

Proof. Let $A$ be the regular open set $S(e)$. First, note that if $P$ is a regular open subset of $X$, then $A \cap P$ is a regular open subset of $A$ and $\operatorname{ext}_{A}(A \cap P)=A \cap \operatorname{ext}_{X} P$. Therefore, $T$ 
is a dyadic subbase. Note also that $\operatorname{cl}_{A}(A \cap P)=A \cap \mathrm{cl}_{X} P$. Therefore, for $d \in \mathbb{T}^{*}$, we have

$$
\begin{aligned}
\operatorname{cl}_{A} \bigcap_{k \in \operatorname{dom}(d)} T_{k, d(k)} & =\operatorname{cl}_{A}\left(A \cap \bigcap_{k \in \operatorname{dom}(d)} S_{\nu(k), d(k)}\right) \\
& =A \cap \operatorname{cl}_{X} \bigcap_{k \in \operatorname{dom}(d)} S_{\nu(k), d(k)} \\
& =A \cap \bigcap_{k \in \operatorname{dom}(d)} \operatorname{cl}_{X} S_{\nu(k), d(k)} \\
& =\bigcap_{k \in \operatorname{dom}(d)}\left(A \cap \operatorname{cl}_{X} S_{\nu(k), d(k)}\right) \\
& =\bigcap_{k \in \operatorname{dom}(d)} \operatorname{cl}_{A}\left(A \cap S_{\nu(k), d(k)}\right) \\
& =\bigcap_{k \in \operatorname{dom}(d)} \operatorname{cl}_{A} T_{k, d(k)} .
\end{aligned}
$$

Therefore, $T$ is proper.

Proposition 5.10. Suppose that $X$ is a strongly nonadhesive space and $S$ is a proper dyadic subbase of $X$.

(1) The poset $K_{S}$ is finite-branching.

(2) The poset $\widehat{K}_{S}$ is finite-branching.

Proof.

(1) Let $e \in \mathbb{T}^{*}$. By applying Proposition 5.8 to the proper dyadic subbase $T$ on $S(e)$ in Lemma 5.9, $\operatorname{succ}(\perp)$ is finite in the poset $K_{T}$. Since $K_{T}$ is identical to $\uparrow e$ in $K_{S}, \operatorname{succ}(e)$ is finite in $K_{S}$.

(2) In this proof, $\operatorname{succ}(d)$ for $d \in K_{S}$ means $\operatorname{succ}(d)$ in $K_{S}$. Let $e \in \widehat{K}_{S}$ and let $k$ be the maximal length of elements in $\cup_{d \in \downarrow e n K_{S}} \operatorname{succ}(d)$, which exists by (1). Suppose that, for some $n \geqslant k$ and $a \in \mathcal{Z}, e[n:=a] \in \widehat{K}_{S}$. Then, for some $x \in X$ and $p \sqsupseteq \tilde{x}$, $\left.p\right|_{n+1}=e[n:=a]$. Therefore, $\left.\tilde{x}\right|_{n} \sqsubseteq e$. For $d_{0}=\left.\tilde{x}\right|_{n}$, let $m>n$ be the least integer such that $\left.\tilde{x}\right|_{m} \ni d_{0}$. The set $\operatorname{succ}\left(d_{0}\right)$ contains $\left.\tilde{x}\right|_{m}$ and we have contradiction.

Theorem 5.11. Suppose that $X$ is a strongly nonadhesive space and $S$ is a proper dyadic subbase of $X$.

(1) $L\left(D_{S}\right)$ has enough minimal elements and $\min \left(L\left(D_{S}\right)\right)$ is compact.

(2) $L\left(\widehat{D}_{S}\right)$ has enough minimal elements and $\min \left(L\left(\widehat{D}_{S}\right)\right)$ is compact.

Proof. From Proposition 5.3 and 5.10 .

Note that, as Proposition 5.5 shows, Theorem 5.11 is applicable to all the Urysohn spaces, in particular, to regular spaces. Note also that the premise of Theorem 5.11 is not a necessary condition for $L\left(D_{S}\right)$ to have enough minimal elements. For example, for the space $A$ and the dyadic subbase $S^{A}$ in Example 5.6, the domain $D_{S^{A}}$ has enough minimal elements and $\varphi_{S^{A}}(A) \subseteq \min \left(L\left(D_{S^{A}}\right)\right)$.

It is shown in 21] that there is a Hausdorff space $X$ and an independent subbase $S$ of $X$ such that $D_{S}$ is equal to $\mathbb{T}^{\omega}$ and therefore $L\left(D_{S}\right)$ does not have enough minimal elements. 


\section{Domain Representations in Minimal-Limit Sets}

Now, we show that $X$ is embedded in $\min \left(L\left(D_{S}\right)\right)$ and $\min \left(L\left(\widehat{D}_{S}\right)\right)$ for the case $S$ is a proper dyadic subbase of a regular space $X$. We start with new notations and a small lemma.

Definition 6.1. For a dyadic subbase $S$ of a space $X, p \in \mathbb{T}^{\omega}$, and $n \in \mathbb{N}$, we define $S_{\mathrm{ex}}^{n}(p) \subseteq X$ and $\bar{S}_{\mathrm{ex}}^{n}(p) \subseteq X$ as follows.

$$
\begin{gathered}
S_{\mathrm{ex}}^{n}(p)=\bigcap_{k<n} S_{k, p(k)}=S\left(\left.p\right|_{n}\right) \cap \bigcap_{\substack{k<n, k \notin \operatorname{dom}(p)}} S_{k, \perp}, \\
\bar{S}_{\mathrm{ex}}^{n}(p)=\bigcap_{k<n} \operatorname{cl} S_{k, p(k)}=\bar{S}\left(\left.p\right|_{n}\right) \cap \bigcap_{\substack{k<n, k \notin \operatorname{dom}(p)}} S_{k, \perp} .
\end{gathered}
$$

Lemma 6.2. Let $e \in \mathbb{T}^{*}$ and $n=|e|$.

(1) $S_{\mathrm{ex}}^{n}(e) \neq \varnothing$ if and only if $e \in K_{S}$.

(2) $\bar{S}_{\mathrm{ex}}^{n}(e) \neq \varnothing$ if and only if $e \in \widehat{K}_{S}$.

Proof.

(1) $x \in S_{\mathrm{ex}}^{n}(e)$ if and only if $e=\left.\tilde{x}\right|_{n}$.

(2) $x \in \bar{S}_{\mathrm{ex}}^{n}(e)$ if and only if $\left.\tilde{x}\right|_{n} \sqsubseteq e$ if and only if there exists $q \sqsupseteq \tilde{x}$ such that $e=\left.q\right|_{n}$.

Theorem 6.3. Suppose that $S$ is a proper dyadic subbase of a regular space $X$ and $D \in$ $\left\{D_{S}, \widehat{D}_{S}\right\}$. If $p \in L(D)$ and $p$ is compatible with $\tilde{x}$ in $\mathbb{T}^{\omega}$, then $p \sqsupseteq \tilde{x}$. In particular, $\tilde{X} \subseteq \min (L(D))$.

Proof. Suppose that $p \in L\left(\mathbb{T}^{\omega}\right)$ satisfies $p \uparrow \tilde{x}$ and $p \neq \tilde{x}$. There is an index $n \in \mathbb{N}$ such that $\tilde{x}(n) \neq \perp$ and $p(n)=\perp$. We assume that $\tilde{x}(n)=0$. That is, $x \in S_{n, 0}$. Since $X$ is regular and $S$ is proper, $x \in S(e) \subseteq \operatorname{cl} S(e)=\bar{S}(e) \subseteq S_{n, 0}$ for some $e \in K\left(D_{S}\right)$. We can assume that $e=\left.\tilde{x}\right|_{m}$ for some $m>n$ such that $p(m-1) \neq \perp$.

We have

$$
\begin{aligned}
& \bar{S}(e)=\bigcap_{k \in \operatorname{dom}(e)}\left(S_{k, e(k)} \cup S_{k, \perp}\right), \\
& \bar{S}_{\mathrm{ex}}^{m}(p)=\bigcap_{\substack{k<m, k \in \operatorname{dom}(p)}}\left(S_{k, p(k)} \cup S_{k, \perp}\right) \cap \bigcap_{\substack{k<m, k \notin \operatorname{dom}(p)}} S_{k, \perp} .
\end{aligned}
$$

Therefore, since $p \uparrow e$, we have $S_{n, 0} \supseteq \bar{S}(e) \supseteq \bar{S}_{\mathrm{ex}}^{m}(p) \supseteq S_{\mathrm{ex}}^{m}(p)$. On the other hand, since $p(n)=\perp$, we have $S_{n, 0} \cap \bar{S}_{\mathrm{ex}}^{m}(p)=\varnothing$. Therefore, we can conclude that both $\bar{S}_{\mathrm{ex}}^{m}(p)=$ $\bar{S}_{\mathrm{ex}}^{m}\left(\left.p\right|_{m}\right)$ and $S_{\mathrm{ex}}^{m}(p)=S_{\mathrm{ex}}^{m}\left(\left.p\right|_{m}\right)$ are empty. Thus, by Lemma 6.2. we have $\left.p\right|_{m} \notin K_{S}$ and $\left.p\right|_{m} \notin \widehat{K}_{S}$. Then, from Lemma 4.7, we have $p \notin D_{S}$ and $p \notin \widehat{D}_{S}$.

Theorem 6.4. Suppose that $S$ is a proper dyadic subbase of a compact Hausdorff space $X$ and $D \in\left\{D_{S}, \widehat{D}_{S}\right\}$. We have $\tilde{X}=\min (L(D))$ and $X$ is a retract of $L(D)$. 
Proof. Since a compact Hausdorff space is regular, we have $\tilde{X} \subseteq \min (L(D))$ by Theorem6.3. Assume that there exists $p \in \min (L(D)) \backslash \tilde{X}$. For every $x \in X, \tilde{x}$ and $p$ are not compatible in $\mathbb{T}^{\omega}$ by Theorem 6.3. Therefore, $\tilde{x}(k)$ and $p(k)$ are different digits for some $k$. Thus, we have an open covering $X=\bigcup_{k \in \operatorname{dom}(p)} S_{k, 1-p(k)}$. Since $\bar{S}\left(\left.p\right|_{m}\right) \neq \varnothing$ for all $m \in \mathbb{N}$, there is no finite subcovering. Therefore, $X$ is not compact.

We state properties of domain representations as a corollary. Here, $\uparrow \tilde{X}$ in $\left(D_{S}, \uparrow \tilde{X}, \rho_{S}\right)$ is the upwards-closure of $\tilde{X}$ in $D_{S}$ which may be different from the upwards-closure of $\widetilde{X}$ in $\mathbb{T}^{\omega}$.

Corollary 6.5. Suppose that $S$ is a proper dyadic subbase of a regular space $X$ and $D \in$ $\left\{D_{S}, \widehat{D}_{S}\right\}$.

(1) In the dense domain representation $\left(D, \tilde{X}, \varphi^{-1}\right)$, we have $\tilde{X} \subseteq \min (L(D))$. In particular, if $X$ is compact, then $\tilde{X}=\min (L(D))$.

(2) In the retract domain representation $\left(D, \uparrow \tilde{X}, \rho_{S}\right), \uparrow \tilde{X}$ is downwards-closed in $L(D)$. In particular, if $X$ is compact, then $\uparrow \widetilde{X}=L(D)$.

Proof. By Theorem 6.3 and 6.4,

As Corollary 6.5 shows, if $X$ is compact, then $\left(D_{S}, L\left(D_{S}\right), \rho_{S}\right)$ and $\left(\widehat{D}_{S}, L\left(\widehat{D}_{S}\right), \rho_{S}\right)$ are representations of $X$ as minimal-limit sets of domains studied in [19. In both of the domains, all the strictly increasing sequences in the set of compact elements denote points of $X$ via $\rho_{S}$.

As we have seen, if $S$ is a proper dyadic subbase of a regular space $X$, then $\min \left(L\left(D_{S}\right)\right)$ is a compact space in which $X$ is embedded densely. Therefore, $\min \left(L\left(D_{S}\right)\right)$ is a kind of compactification of $X$. However, it is not a Hausdorff compactification, in general, as Example 6.6 shows.

Example 6.6. Let $Z=\mathbb{I} \times \mathbb{I}$ be a unit square. An independent subbase $H$ of $Z$ is defined as

$$
H_{2 k, a}=G_{k, a} \times \mathbb{I}, \quad H_{2 k+1, a}=\mathbb{I} \times G_{k, a}, \quad \text { for } k \in \mathbb{N}, a \in \mathcal{Z} .
$$

We have $\varphi_{H}((1 / 2,1 / 2))=\perp \perp 110^{\omega}$. We set $A=\{00 p, 01 p, 10 p, 11 p\}$ and $B=\{0 \perp p, 1 \perp p, \perp 0 p$, $\perp 1 p\}$ where $p=110^{\omega} \in \mathbb{T}^{\omega}$. Note that $\uparrow \perp \perp p=\{\perp \perp p\} \cup A \cup B$.

Let $Z^{0}=\mathbb{I} \times \mathbb{I} \backslash\{(1 / 2,1 / 2)\}$ be a subspace of $Z$. The independent subbase of $Z^{0}$ which is obtained by restricting each element of $H$ to $Z^{0}$ is denoted by $H^{0}$. We have $L\left(D_{H^{0}}\right)=$ $L\left(D_{H}\right) \backslash\{\perp \perp p\}$ and we get

$$
\min \left(L\left(D_{H^{0}}\right)\right)=\left(\min \left(L\left(D_{H}\right)\right) \backslash\{\perp \perp p\}\right) \cup B .
$$

Since the set $B$ contains a pair of compatible bottomed sequences, $\min \left(L\left(D_{H^{0}}\right)\right)$ is not Hausdorff.

Example 6.7. Let $Z^{1}=Z \backslash\{(1 / 2,1 / 3)\}$ be a subspace of $Z$ and $H^{1}$ be a dyadic subbase of $Z^{1}$ defined similarly to Example 6.6. We have

$$
\widetilde{Z}^{1}=\min \left(L\left(D_{H}\right)\right) \backslash\{q\}
$$

where $q=\perp 011(01)^{\omega}$. However, since we have

$$
\left|y-\frac{1}{3}\right|<\left.\frac{1}{3 \cdot 2^{n}} \Rightarrow \varphi_{H^{1}}((1 / 2, y))\right|_{2 n}=\left.q\right|_{2 n}
$$


for all $n \in \mathbb{N}$, we get $L\left(D_{H^{1}}\right)=L\left(D_{H}\right)$. Therefore $\min \left(L\left(D_{H^{1}}\right)\right)$ is a Hausdorff compactification of $Z^{1}$.

Example 6.8. We set $Z^{2}=Z^{1} \cup\left\{x_{0}, x_{1}\right\}$ with $\tilde{x}_{a}=q[0:=a]$ for $q$ in Example 6.7$]$ and $a \in \mathcal{Z}$, and let $H^{2}$ be the corresponding dyadic subbase. The space $Z^{2}$ is a non-regular Hausdorff space and we have $\min \left(L\left(D_{H^{2}}\right)\right)=\min \left(L\left(D_{H}\right)\right)$. Since we have $\tilde{x}_{a} \notin \min \left(L\left(D_{H^{2}}\right)\right)$, we get $\widetilde{X} \nsubseteq \min \left(L\left(D_{H^{2}}\right)\right)$.

\section{Height of $L\left(D_{S}\right)$ And the dimension of $X$}

We finally study relations between the degree of a proper dyadic subbase $S$ and structures of $L\left(D_{S}\right)$ and $L\left(\widehat{D}_{S}\right)$.

Definition 7.1. For a dyadic subbase $S$ of a space $X$ and $x \in X$, we define $\operatorname{deg}_{S}(x)=\mid\{n \in$ $\left.\mathbb{N}: x \in S_{n, \perp}\right\} \mid$ and $\operatorname{deg} S=\sup \left\{\operatorname{deg}_{S}(x): x \in X\right\}$.

If $\operatorname{deg} S=m$, then $\varphi_{S}(x)$ contains at most $m$ copies of $\perp$ for $x \in X$. It is proved in [11] that every separable metrizable space $X$ with $\operatorname{dim} X=m$ has a proper dyadic subbase $S$ with $\operatorname{deg} S=m$. Here, $\operatorname{dim} X$ is the covering dimension of $X$. It is known that $\operatorname{dim} X$ is equal to the small inductive dimension ind $X$ of $X$ for a separable metrizable space $X$. See, for example, [5] and [6] for dimension theory.

For a domain $D$, we consider the small inductive dimension ind $L(D)$ of the topological space $L(D)$ with the subspace topology of the Scott topology of $D$. In Theorem 6.11 of [19], it is proved that if $D$ is a domain with property $\mathrm{M}$, then ind $L(D)=$ height $L(D)$ holds. Here, height $P$ is the maximal length of a chain $a_{0} \sqsubset a_{1} \sqsubset \ldots \sqsubset a_{n}$ in a poset $P$. Property $\mathrm{M}$ is defined as follows.

Definition 7.2. (1) We say that a poset $P$ is mub-complete if for every finite subset $A \subseteq P$, the set of upper bounds of $A$ has enough minimal elements. That is, if $p$ is an upper bound of $A$, then there exists a minimal upper bound $q$ of $A$ such that $q \sqsubseteq p$.

(2) We say that a domain $D$ has property $M$ if $K(D)$ is mub-complete and each finite subset $A \subseteq K(D)$ has a finite set of minimal upper bounds.

Property $M$ is equivalent to Lawson-compactness for $\omega$-algebraic depo by the $2 / 3 \mathrm{SFP}$ Theorem [13. Domains with property $M$ are studied in [10].

Proposition 7.3. Suppose that $S$ is a proper dyadic subbase of a regular space $X$.

(1) The domains $D_{S}$ and $\widehat{D}_{S}$ have property $M$.

(2) ind $L\left(\widehat{D}_{S}\right)=$ height $L\left(\widehat{D}_{S}\right) \geqslant$ ind $L\left(D_{S}\right)=\operatorname{height} L\left(D_{S}\right) \geqslant \operatorname{dim} X$.

(3) If $X$ is compact, then ind $L\left(\widehat{D}_{S}\right)=\operatorname{deg} S$.

Proof.

(1) Since bounded completeness implies property $\mathrm{M}, \widehat{D}_{S}$ has property M. For $D_{S}$, suppose that a finite subset $A \subseteq K_{S}$ has an upper bound. Let $d$ be the least upper bound of $A$ in $\mathbb{T}^{*}$. Then, $e \in K_{S}$ is an upper bound of $A$ in $K_{S}$ if and only if $e \sqsupseteq d$. If $d \in K_{S}$, then it is the only minimal upper bound of $A$. Suppose that $d \notin K_{S}, e \sqsupseteq d$, and $e \in K_{S}$. Then, for $n=|d|,\left.e\right|_{n} \sqsupseteq d$ and $\left.e\right|_{n} \in K_{S}$ by Lemma 4.7(1). Therefore, if $e$ is a minimal upper bound of $A$, then $e=\left.e\right|_{n}$ and the length of $e$ is no more than $n$. Therefore, the set of minimal upper bounds of $A$ is finite. 
(2) The equation ind $L(D)=$ height $L(D)$ for $D \in\left\{D_{S}, \widehat{D}_{S}\right\}$ is derived from (1) and Theorem 6.11 of [19]. We have ind $L\left(D_{S}\right) \geqslant$ ind $X$ because $X$ is embedded in $L\left(D_{S}\right)$ and ind $X=\operatorname{dim} X$ for a separable metrizable space $X$.

(3) Since $\uparrow \tilde{x}$ in $\hat{D}_{S}$ and $\uparrow \tilde{x}$ in $\mathbb{T}^{\omega}$ are the same set for $x \in X$, the maximum number of bottoms in $\tilde{x}$ for $x \in X$ is equal to the height of $L\left(\widehat{D}_{S}\right)$, which is equal to the small inductive dimension of $L\left(\widehat{D}_{S}\right)$ by $(2)$.

Note that ind $L\left(D_{S}\right)$ may not be equal to $\operatorname{deg} S$ even for an independent subbase of a compact space $X$ as Example 4.3 shows. In this example, the height of $L\left(D_{R}\right)$ is one, whereas that of $L\left(\widehat{D}_{R}\right)$ is two.

\section{ACKNOWLEDGEMENT}

The authors thank anonymous referees for careful readings and many valuable comments.

\section{REFERENCES}

[1] S. Abramsky and A. Jung. Domain theory. In Handbook of Logic in Computer Science, volume 3, pages 1-158. Oxford University Press, 1994.

[2] U. Berger. Total sets and objects in domain theory. Ann. Pure Appl. Logic, 60(2):91-117, 1993.

[3] J. Blanck. Domain representations of topological spaces. Theoretical Computer Science, 247:229 - 255, 2000.

[4] V. Brattka and P. Hertling. Topological properties of real number representations. Theoretical Computer Science, 284(2):241-257, 2002.

[5] R. Engelking. General topology. Monografie matematyczne. PWN, 1977.

[6] R. Engelking. Theory of dimensions, finite and infinite. Sigma series in pure mathematics. Heldermann Verlag, 1995.

[7] P. D. Gianantonio. An abstract data type for real numbers. Theoritical Computer Science, 221:295-326, 1999.

[8] G. Gierz, K. H. Hofmann, K. Keimel, J. D. Lawson, M. W. Mislove, and D. S. Scott. Continuous Lattices and Domains. Cambridge University Press, 2003.

[9] G. Hamrin. Effective Domains and Admissible Domain Representations. Ph.D. thesis, Uppsala dissertations in mathematics 42, Uppsala University, 2005.

[10] A. Jung. Cartesian Closed Categories of Domains, volume 66 of CWI Tracts. Centrum voor Wiskunde en Informatica, Amsterdam, 1989.

[11] H. Ohta, H. Tsuiki, and K. Yamada. Every second-countable regular space has a proper dyadic subbase, 2013. http://arxiv.org/abs/1305.3393.

[12] H. Ohta, H. Tsuiki, and S. Yamada. Independent subbases and non-redundant codings of separable metrizable spaces. Topology and its applications, 158:1-14, 2011.

[13] G. D. Plotkin. Post-graduate lecture notes in advanced domain theory (incorporating the "Pisa Notes"). Technical report, Department of Computer Science, University of Edinburgh, 1981.

[14] M. Schröder. Extended admissibility. Theoretical Computer Science, 284(2):519-538, 2002.

[15] V. Stoltenberg-Hansen, I. Lindström, and E. Griffor. Mathematical Theory of Domains. Cambridge University Press, 1994.

[16] V. Stoltenberg-Hansen and J. Tucker. Effective algebra. In S. Abramsky et al., editors, Handbook of Logic in Computer Science, volume 4, pages 357-526. Oxford University Press, 1995.

[17] V. Stoltenberg-Hansen and J. V. Tucker. Computability on topological spaces via domain representations. In New Computational Paradigms, pages 153-194. Springer, 2008.

[18] H. Tsuiki. Real number computation through gray code embedding. Theoretical Computer Science, 284(2):467-485, 1977.

[19] H. Tsuiki. Compact metric spaces as minimal-limit sets in domains of bottomed sequences. Mathematical Structures in Computer Science, 14(6):853-878, 2004. 
[20] H. Tsuiki. Dyadic subbases and efficiency properties of the induced $\{0,1, \perp\}^{\omega}$-representations. Topology Proceedings, 28(2):673-687, 2004.

[21] Y. Tsukamoto and H. Tsuiki. Properties of domain representations of spaces through dyadic subbases Mathematical Structures in Computer Science, to appear.

[22] K. Weihrauch. Computable analysis: an introduction. Springer-Verlag New York, Inc., Secaucus, NJ, USA, 2000.

[23] K. Weihrauch and T. Grubba. Elementary computable topology. Journal of Universal Computer Science, 15(6):1381-1422, 2009. 